\title{
Comparison of periodic and random structures for scattering in thin- film microcrystalline silicon solar cells
}

\author{
M. Peters ${ }^{\mathrm{a}, \mathrm{b}}$, K. Forberich ${ }^{\mathrm{a}}$, C. Battaglia ${ }^{\mathrm{c}}$, A. G. Aberle ${ }^{\mathrm{a}}$, B. Bläsis ${ }^{\mathrm{b}}$ \\ ${ }^{a}$ Solar Energy Research Institute of Singapore (SERIS), 7 Engineering Drive 1, Singapore 117574, \\ Singapore \\ ${ }^{\mathrm{b}}$ Fraunhofer Institute for Solar Energy Systems (ISE), Heidenhofstrasse 2, 79110 Freiburg, \\ Germany \\ ${ }^{\mathrm{c}}$ Ecole Polytechnique Federale de Lausanne (EPFL), 2000 Neuchâtel, Switzerland
}

\begin{abstract}
Random structures are typically used for light trapping in thin-film silicon solar cells. However, theoretically periodic structures can outperform random structures in such applications. In this paper we compare random and periodic structures of similar shape. Both types of structure are based on atomic force microscopy (AFM) scans of a sputtered and etched $\mathrm{ZnO}$ layer. The absorption in a solar cell on both structures was calculated and compared to external quantum efficiency (EQE) measurements of samples fabricated on the random texture. Measured and simulated currents were found to be comparable. A scalar scattering approach was used to simulate random structures, the rigorous coupled wave analysis (RCWA) to simulate periodic structures. The length and height of random and periodic structures were scaled and changes in the photocurrent were investigated. A high height/length ratio seems beneficial for periodic and random structures. Very high currents were found for random structures with very high roughness. For periodic structures, current maxima were found for specific periods and heights. An optimized periodic structure had a period of $\Lambda=534 \mathrm{~nm}$ and a depth of $d=277 \mathrm{~nm}$. The photocurrent of this structure was increased by $1.6 \mathrm{~mA} / \mathrm{cm}^{2}$ or $15 \%$ relative compared to the initial (random) structure in the spectral range between $600 \mathrm{~nm}$ and $900 \mathrm{~nm}$.
\end{abstract}

Keywords: Thin-film microcrystalline silicon solar cells, diffraction, scattering.

\section{INTRODUCTION}

Efficient light trapping is of key importance for thin-film crystalline silicon solar cells. The most common approach to achieve light trapping for this kind of solar cell is to use a scattering texture. Scattering structures are typically nonperiodic, randomly textured surfaces onto which the solar cell system is deposited. The theoretical maximum in absorption enhancement that can be achieved with a scattering structure is given by the Yablonovitch factor $4 \mathrm{n}^{2}$ [1] with $\mathrm{n}$ the refractive index of the absorber material. For silicon with a refractive index of $\mathrm{n}=3.5$, the Yablonovitch factor is close to 50 . The effectiveness of scattering textures has been shown on many occasions and high solar cell efficiencies are achieved with these structures [2,3]. However, in many cases the absorption enhancement is still significantly lower than the Yablonovitch factor.

Another promising approach is light trapping with periodic, diffractive structures (gratings or photonic crystals). This concept was first proposed by Sheng [4] for binary linear gratings for thin-film solar cells and has later been extended to other types of solar cells and gratings [5-8]. It has been shown on several occasions that diffractive gratings can result in a pathlength enhancement which exceeds the Yablonovitch factor. The theoretical maximum that can be achieved with such a structure is an absorption enhancement of $853 n$ [9] with $\mathrm{n}$ again the refractive index. For silicon, a factor of approximately 3000 is obtained. This value is based on thermodynamical arguments and marks the theoretical limit. Recently, different 2D gratings have been proposed for which simulations showed a pathlength enhancement exceeding the Yablonovitch factor $[10,11]$. These structures however, show a very complex geometry and the realization of large scale samples is an open point.

At this point in time, the discussion whether periodic or non-periodic structures are superior has not come to a conclusion. It is widely believed that periodic textures have the higher potential, but they also will increase the

Photonics for Solar Energy Systems IV, edited by Ralf Wehrspohn, Andreas Gombert

Proc. of SPIE Vol. 8438, 84380F · C 2012 SPIE · CCC code: 0277-786X/12/\$18 · doi: 10.1117/12.921774 
production costs because they require at least an additional masking step. Furthermore, it is very difficult to tell how an optimum diffractive structure will look like because of the numerous possibilities of shape and geometry. In this paperwe chose to investigate structures that can be realized experimentally, rather than to look for an optimum geometry that might be very difficult to realize for a real solar cell. The idea is to compare periodic and aperiodic structures that are similar in shape. For this purpose we start with analyzing the surface of a scattering texture and identify typical single craters that were produced in the etching process. These craters will have a realistic shape and it can be assumed that they can be produced with an established texturization process. In a second step we numerically construct a periodic continuum of the single craters. This texture could be realized using a simple masking process and using a similar etching process as was used to create the non-periodic texture. This process and the investigated structures are described in the following chapter. To estimate the optical absorption that can be expected in the solar cell from the periodic and non-periodic texture, we use different simulation tools. For periodic structures we use the rigorous coupled wave analysis (RCWA), for non-periodic structures we use an approach based on scalar scattering theory (SST). A short introduction into the used simulation tools is given in chapter 3. Results are presented in chapter 4 . The paper concludes with a summary and discussion of the results.

\section{DETAILS OF INVESTIGATED STRUCTURES}

Our investigation is based on an AFM measurement of a sputtered and subsequently etched $\mathrm{ZnO}$ texture. The texture is shown in Figure 1. The applied etching process results in a structure of craters with random size but similar shapes. Light impinging on this structure is scattered into a certain angular distribution and trapped accordingly. A theoretical investigation and comparison to measurements of the light trapping properties of this structure are shown in section 4.1.

To compare the light trapping of this random structure to a periodic structure of similar shape, we performed a structure analysis with the method described in Ref. [12]. Using this analysis, various typical shapes occurring in the random structure were identified. In a first screening, the absorption in a solar cell structure on these textures was calculated. The solar cell structure consists of a glass superstrate with a textured $\mathrm{ZnO}$ film $(1.8 \mu \mathrm{m}, n=2.0)$, the active $\mu \mathrm{c}$-Si solar cell $(1.1 \mu \mathrm{m}, n$ measured at EPFL) another ZnO layer and a back reflector. In this investigation, silicon is considered to be the only absorbing material; for all other materials we assumed $k=0$ for all wavelengths. From the structure analysis, the parameter set for the best performing crater structure with the highest photocurrent was chosen as basis for the present study. The chosen periodic structure had a period of $\Lambda=763 \mathrm{~nm}$ and a depth of $d=252 \mathrm{~nm}$. It is displayed in Figure 1 on the right hand side. A theoretical investigation of the light trapping that can be expected from this structure is presented in section 4.2 .

In addition to the contour plots, also cross sections along the red lines shown in Figure 1 are displayed. In the cross section for the periodic structures (right hand side) the defining parameters of the periodic structure period $(\Lambda)$ and depth (d) are shown.

a) sketch of aperiodic structure

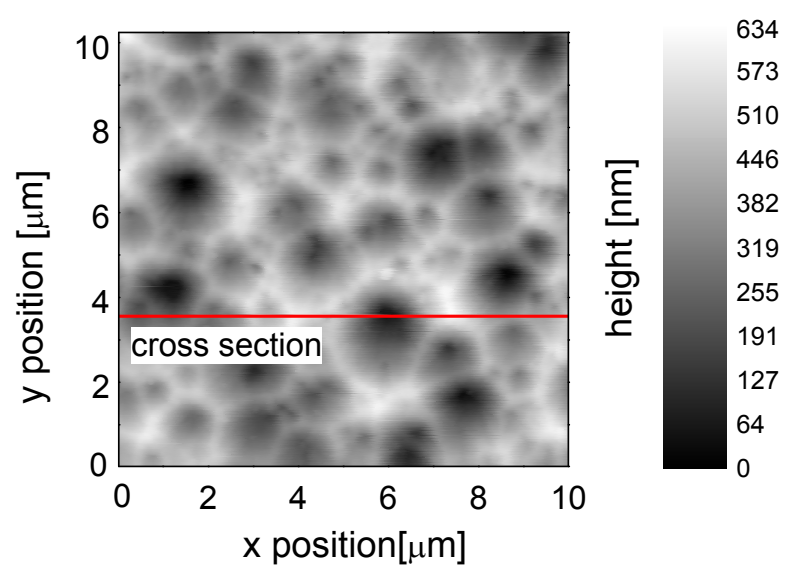

b) sketch of periodic structure

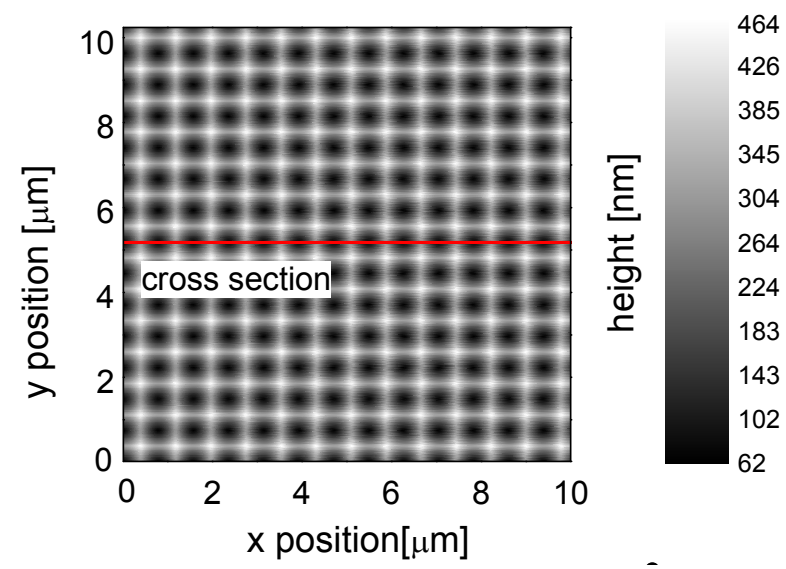


c) height profile random structure

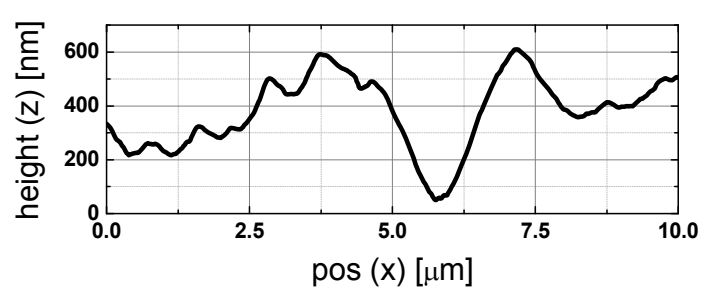

d) height profile periodic structure

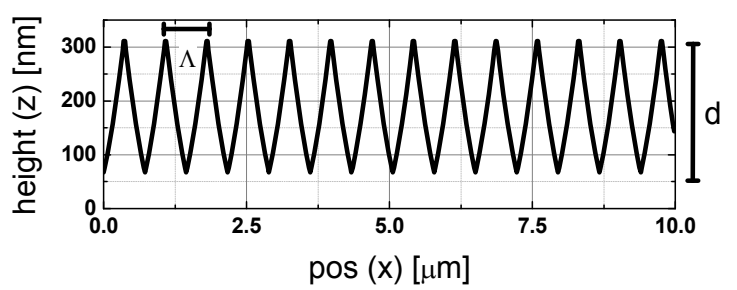

Figure 1 The contour plots of an AFM scan of the random texture used for this study is shown in Figure 1a.. The texture consists of a sputtered and etched $\mathrm{ZnO}$ layer. Also shown is a cross section along the red line through this scan (Figure 1c). Figure $1 \mathrm{~b}$ shows the contour plot of a periodic structure constructed from the $\mathrm{ZnO}$ texture is given. In Figure 1d, a cross section through this periodic structure is displayed including the main parameters for the periodic structure period $(\Lambda)$ and depth $(d)$

\section{SIMULATION METHODS}

\section{Rigorous Coupled Wave Analysis (RCWA)}

The rigorous coupled wave analysis is a rigorous method to solve Maxwell's equations. It is based on a Fourier transformation of both the electromagnetic field and the structure. The structure is described as a function of the complex refractive index in space. The refractive index is included in Maxwell's equations and the Fourier transformation allows writing the solutions of a given problem in a sum. The summands can be interpreted to be plane waves describing the orders of diffraction for the investigated structure. Solutions inside the structure are found by first solving the problem for the hemispheres of incidence and transmission and subsequently constructing the field in the structure by superimposing these solutions. Further details on the method can be found in Ref. [13]. The implementation used in this work was that of Lalanne and Jurek [14].

The RCWA is used to calculate near- and far-field properties of the investigated structure. In the presented study, we show only far field properties. We use the RCWA to calculate the optical absorption $a b s(\lambda)=1-R-T$ within the active solar cell material in the structure. From the absorption we calculate the corresponding absorbed photocurrent density $j_{p h}$ via

$$
j_{p h}=\int_{\lambda_{1}}^{\lambda_{2}} a b s(\lambda) N_{A M 1.5}(\lambda) d \lambda
$$

In this equation $N_{A M 1.5}(\lambda)$ is the photon flux of the AM1.5G solar spectrum $\left(1000 \mathrm{~W} / \mathrm{m}^{2}\right)$. In this paper, $j_{p h}$ was calculated for a spectral range between $600 \mathrm{~nm}$ and $900 \mathrm{~nm}$ in steps of $10 \mathrm{~nm}$. Within this spectral range, the structure has the highest absolute effect on the absorbed photocurrent; The thickness already limits the number of photons absorbed while the number of photons in the spectrum is still comparably high. It can be assumed that this spectral range covers most of the gain in current generation. The wavelength range was not extended further, also because RCWA calculations are very time-consuming. It is important to note that $j_{p h}$ marks an upper limit but is principally different from the shortcircuit current density $j_{S C}$, which is calculated by

$$
j_{S C}=\int_{\lambda_{1}}^{\lambda_{2}} \operatorname{abs}(\lambda) \operatorname{IQE}(\lambda) N_{A M 1.5}(\lambda) d \lambda=\int_{\lambda_{1}}^{\lambda_{2}} \operatorname{EQE}(\lambda) N_{A M 1.5}(\lambda) d \lambda
$$

In this equation $\operatorname{IQE}(\lambda)$ is the internal quantum efficiency and $\operatorname{EQE}(\lambda)$ is the external quantum efficiency of the investigated solar cell. The IQE includes all electronic losses that occur during current generation and collection. The IQE for different crystalline thin-film silicon solar cells can be assumed to be in the range of $90 \%[15,16]$.

It needs to be said that the presented RCWA results are not yet verified by measurements of actual fabricated periodic structures and are therefore to some extent speculative. This must be kept in mind when comparing the simulated results obtained by RCWA and the presented measured results. 


\section{Scalar Scattering Theory (SST)}

We used a scalar approach that was proposed, for example, in Refs. [2] and [17]. From a measured AFM scan, we can calculate pupil functions in transmittance and reflectance

$$
G_{R}=e^{i k_{0} \zeta(x, y) 2 n_{1}} \text { and } G_{T}=e^{i k_{0} \zeta(x, y)\left(n_{1}-n_{2}\right)},
$$

where $T$ and $R$ denote transmission and reflection, respectively, $k_{0}$ stands for the vacuum wave vector of the incident light, $n_{1}$ and $n_{2}$ are the refractive indices of the incoming and outgoing medium and $\zeta(x, y)$ is the measured height of the scattering surface. We then obtain the angular distribution of the transmitted and reflected light from the Fourier transform of the respective pupil function. Haze values are calculated according to

$$
H_{R}=1-e^{-\left(\frac{4 \pi \sigma_{r m s} n_{1}}{\lambda}\right)^{2}} \text { and } H_{T}=1-e^{-\left(\frac{2 \pi \sigma_{r m s}\left(n_{1}-n_{2}\right)}{\lambda}\right)^{2}}
$$

where $\sigma_{r m s}$ is the rms roughness as determined from the AFM scan and $\lambda$ is the wavelength. Haze and angular distribution function are then used as input parameters for the ASA opto-electrical device simulator [18] to calculate the absorbance in the silicon layer. The quantities $j_{p h}$ and $j_{S C}$ are calculated in the same way as described above in section 3.1.

\section{RESULTS}

\subsection{Optimization of the random structure}

As a first step, we performed a calculation of the absorptance in the silicon layer for the structure described in section 1.1 and following the approach described in section 2.2. Fig. 2 shows the calculated values and the measured EQE in the wavelength range between $600 \mathrm{~nm}$ and $900 \mathrm{~nm}$. The EQE was measured at IMT Neuchatel for a p-i-n microcrystalline silicon solar cell deposited by plasma-enhanced chemical vapor deposition in an industrial KAI reactor with parallel plate configuration at $180^{\circ} \mathrm{C}$ on the substrate shown in Figure 1a. The solar cell has been characterized using a with a dual lamp sun simulator in standard test conditions $\left(25^{\circ} \mathrm{C}\right.$, global air mass 1.5 (AM1.5G) spectrum, $\left.1000 \mathrm{Wm}^{-2}\right)$. The cell results are given in Table 1. Open-circuit voltage $\left(V_{O C}\right)$ and fill factor $(F F)$ were calculated directly from these measurements, The short-circuit current density $\left(j_{S C}\right)$ was determined by convolution of the EQE and the incoming photon flux of the AM1.5G spectrum.

Table 2 Characteristics of the solar cell used in this study.

\begin{tabular}{|c|c|c|c|}
\hline$V_{O C}(\mathrm{mV})$ & $j_{S C}\left(\mathrm{~mA} \mathrm{~cm}^{-2}\right)$ & $F F(\%)$ & $\eta(\%)$ \\
\hline 540 & 20.4 & 74 & 8.2 \\
\hline
\end{tabular}

Assuming an IQE of $90 \%$ over the entire investigated spectral range, the values are in very good agreement. The reason to compare the simulated absorption to the measured $\mathrm{EQE}$ is that for measured absorption data, the desired absorption in silicon cannot be separated from parasitic absorption in other parts of the solar cell system. Typically, the measured absorption is still quite large even for wavelengths close to the band edge of the solar cell material. The EQE is therefore a much better measure of how much light is actually utilized within the electrically active material than the absorption. 


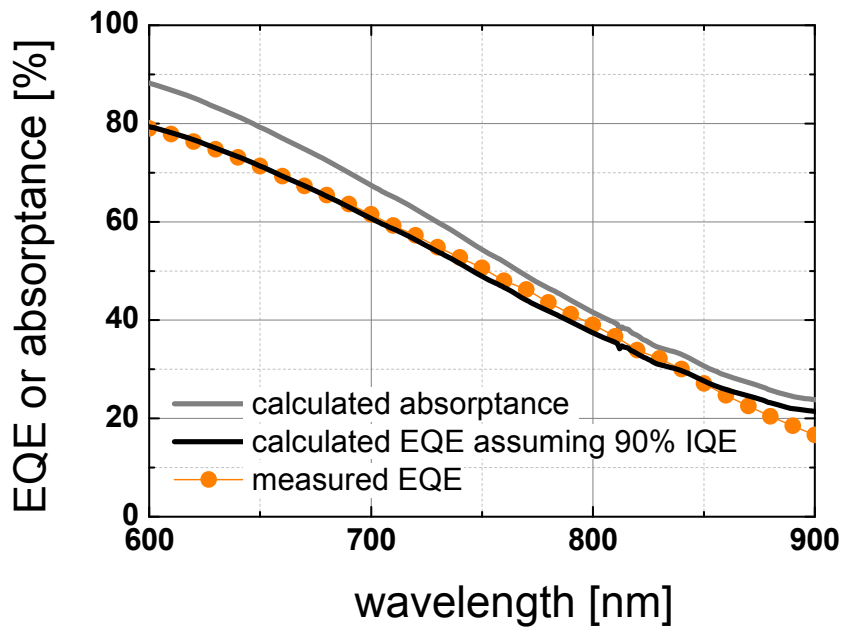

Figure 2. Absorptance in the silicon layer, calculated using the scalar approach and ASA. Assuming an IQE of 90\%, we get a very good agreement between the measured and the simulated data. Both measured and calculated EQE correspond to a photocurrent of $j_{S C}=10.5 \mathrm{~mA} / \mathrm{cm}^{2}$ for this wavelength rang. EQE data was measured at IMT Neuchatel.

We also performed a structure optimization by artificially changing the height and the length scale of the AFM scan shown in Fig.1a. A similar approach has been used for a-Si:H solar cells in Ref [20] The resulting photocurrent density $j_{p h 600-900}$ is shown in Fig. 3. For this optimization, only one interface, the interface between the first TCO and the silicon layer, was assumed to be textured. In a real solar cell device, scattering occurs at each textured interface and it can be assumed that multiple scattering will work more efficiently than scattering at a single interface. Considering only one interface at which light is scattered is therefore a simplification. The general trends should, however, be reproducible even with this simple approach. Moreover, scattering between TCO and silicon can be assumed to be the most important scattering mechanism for the absorption enhancement in the active solar cell material.

First tests with the simulation of multi interface scattering were performed but we are so far not able to create consistent results. Problems here could originate from omitting multiple scattering at the same interface and from the calculation of the reflected and transmitted intensities for subsequently scattered light.

From Fig. 3 we see that an increased height leads to an increase in $j_{p h} 600-900$, which can be explained by the higher $r m s$ value and the consequently higher haze value (Eq. 4). Equivalently, a decrease in height leads to a decrease in $j_{p h} 600-900$. It has to be noted that eq. 4 were derived for small aspect ratios, and that the haze values and therefore the calculated $j_{p h} 600$ 900 tend to be overestimated for large aspect ratios.

A smaller length is equivalent to higher periods and leads to scattering into higher angles, therefore we observe an increase in $j_{p h} 600-900$, and vice versa. This result corresponds to the expectation that a rougher (small length, bigheight, lower right corner in Fig 3) structure should result in a more efficient scattering. A slight increase in the calculated current for very big scaling factors of the length is assumed to be an artifact of the calculation that occurs for certain distributions of very small angles. Additionally we have drawn a line which represents all points for a constant length/height (aspect) ratio. Unless the scaling factors are very small it can be seen that for a constant aspect ratio the current is roughly constant. For very high aspect ratios, the calculated current reaches a maximum of $j_{p h}=17.4 \mathrm{~mA} / \mathrm{cm}^{2}$, which is not too far from the calculated Lambertian limit for this case of $j_{p h}=18.5 \mathrm{~mA} / \mathrm{cm}^{2}$. 


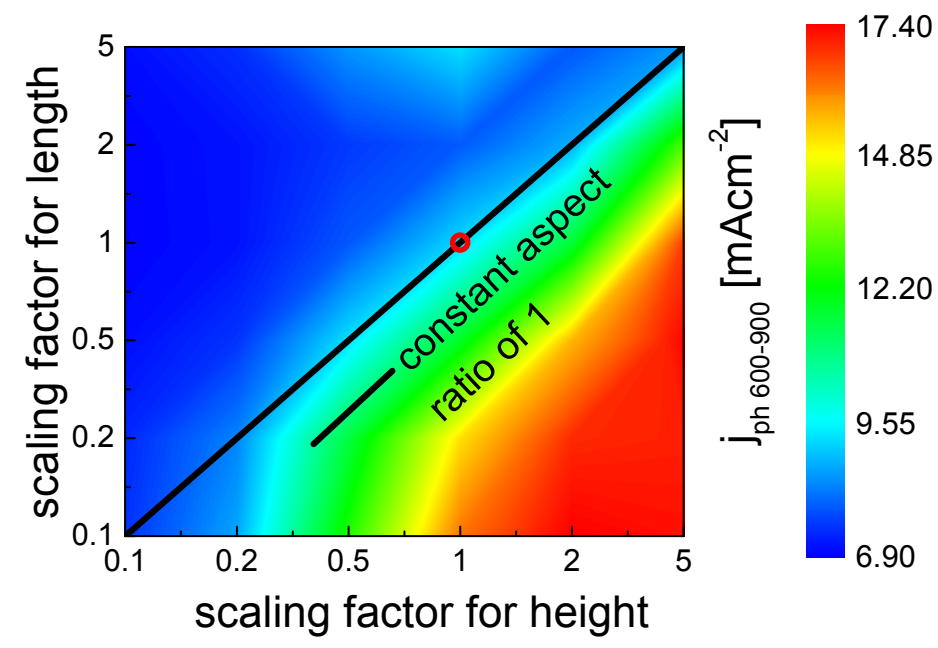

Figure 3. Photocurrent density between $600 \mathrm{~nm}$ and $900 \mathrm{~nm} j_{p h} 600-900$, calculated for a variation of the height and the length scale of the original AFM scan shown in Fig. 1a using the scalar approach and ASA. The original height and the original length were multiplied with the factors given for the axes labeling. The red dot marks the initial situation, the black line marks a constant aspect length to height ratio of 1 .

\subsection{Optimization of the periodic structure using RCWA}

To optimize the identified periodic structure we performed a variation of the period $\Lambda$ and the depth $d$ of the investigated structure (Figure 1,right). The variation was performed by scaling both quantities with a factor $s_{\Lambda}$ and $s_{d}$. The scaling factors were varied between 0.6 and 1.2 in steps of 0.1. Note that these scaling factors span a much smaller range than for the random structure. This is because RCWA calculations are much more time consuming, especially for large periods, than SST calculations. The result of this variation is shown in Figure 4. The red circle marks the initial point of the optimization. The parameters here correspond to the initial situation as shown in Figure 4. The black line marks a constant aspect ratio of 1 of the structure. It can be assumed that these structures can be realized by adjusting the period $\Lambda$ of the etching mask. Moving to the lower right in Figure 4, the aspect ratio is increased, to the upper left, it is reduced. For a constant aspect ratio we find only a small variation of the absorbed current., The estimated short-circuit current in the investigated spectral range lies between 10 and $11 \mathrm{~mA} / \mathrm{cm}^{2}$ which is close to the value obtained from EQE measurements of $10.5 \mathrm{~mA} / \mathrm{cm}^{2}$. As before, $j_{S C}$ is estimated using Eq. 2 from absorption values obtained from RCWA and assuming a $90 \%$ IQE.

Similar to the case of the random structure, we find the trend of higher currents for higher aspect ratios (lower right) and lower currents for lower aspect ratios (upper left). However, a correlation of current to aspect ratio as it was found for the scattering structure cannot be found here. Rather, as can be expected for diffractive structures, specific maxima and minima exist for a specific set of parameters. The position of maxima and minima seems more sensitive on the period than on the depth of the structure. In the investigated parameter range, the highest current is found for a period of $\Lambda=$ $534 \mathrm{~nm}$ and a depth of $d=277 \mathrm{~nm}$. For this optimized structure we find an absorbed photocurrent density of $j_{p h} 600-900=$ $13.3 \mathrm{~mA} / \mathrm{cm}^{2}$ corresponding to an estimated short-circuit current density of $j_{S C 600-900}=12.1 \mathrm{~mA} / \mathrm{cm}^{2}$. This marks an improvement of $\Delta j_{p h}=1.6 \mathrm{~mA} / \mathrm{cm}^{2}$ or $15 \%$ relative in the investigated spectral range. Within the investigated parameter range, this improvement exceeds the one that was calculated for a scattering structure. What happens for much bigger aspect ratios is currently unclear and is subject to future work. However, neither RCWA nor SST might be the appropriate method for calculating structures with extreme parameter sets and this investigation shall rather highlight the trends.

On the other hand, for a period of $\Lambda=687 \mathrm{~nm}$ and a small depth $(d=174 \mathrm{~nm})$, the absorbed photocurrent is as low as $j_{p h}$ ${ }_{600-900}=9.3 \mathrm{~mA} / \mathrm{cm}^{2}$, corresponding to an estimated short circuit current density of $j_{S C 600-900}=8.5 \mathrm{~mA} / \mathrm{cm}^{2}$. This value is however still considerably higher than for comparable values of the random structure. 


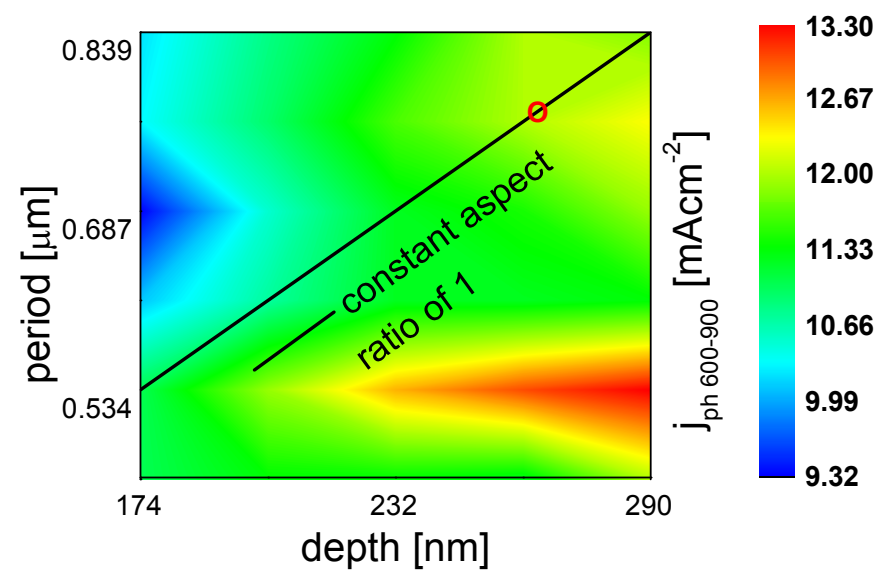

Figure 4.Calculated absorbed photocurrent density $j_{p h}$ for a variation of the parameters $\Lambda$ and $d$ using RCWA. The red circle marks the parameter set for the initial structure (Figure 1). The black line marks all structures with a constant aspect ratio. The highest value of is obtained for a parameter set of $\Lambda=534 \mathrm{~nm}$ and $d=277 \mathrm{~nm}$.

The external quantum efficiency (EQE) of a solar cell with a planar surface (no texture) but otherwise the same setup as for the textured surface was also measured at IMT Neuchatel. The result of this measurement is shown in Figure 5 (left, squares). In the given spectral range $(600-900 \mathrm{~nm})$, this quantum efficiency corresponds to a short-circuit current density of $\mathrm{j}_{\mathrm{SC} 600-900}=7.4 \mathrm{~mA} / \mathrm{cm}^{2}$. Also shown in this graph is the absorption simulated for the planar setup using the transfer matrix method (TMM) [19] (black line). Absorption in the TCO is omitted, which results trend wise in an overestimation of the absorbed current at shorter wavelengths. Fabry Perot oscillations were reduced by allowing partial incoherence, which was realized by performing a small variation of the absorber layer thickness ( $50 \mathrm{~nm}$ in 10 steps) and averaging over the results. With this procedure, a good agreement between the simulated absorption and the measured quantum efficiency is found. Assuming that the solar cell has a non-perfect internal quantum efficiency, the measurement points towards a little higher thickness of the absorber layer than the assumed $1.1 \mu \mathrm{m}$. The simulated absorption results in an absorbed photocurrent of $\mathrm{j}_{\text {ph600-900 }}=7.7 \mathrm{~mA} / \mathrm{cm}^{2}$ and, again using the $90 \%$ estimation of the IQE a generated current of about $7 \mathrm{~mA} / \mathrm{cm}^{2}$.

a) planar

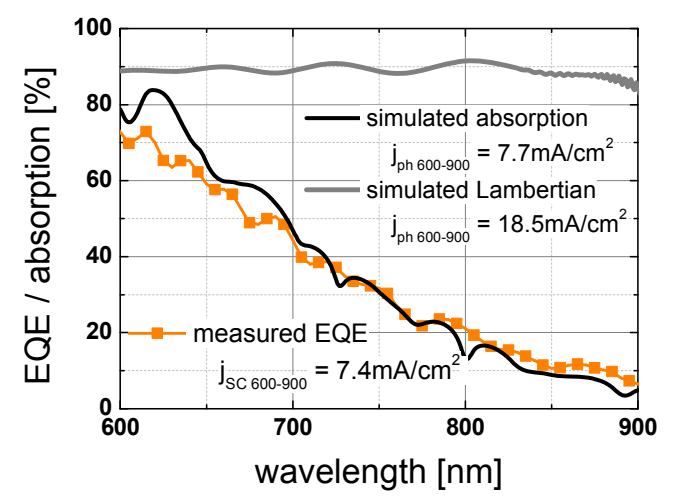

b) textured

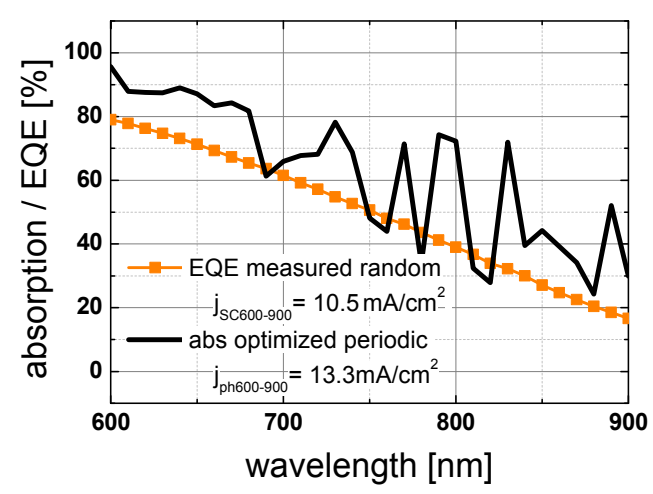

Figure 5.Simulated and measured results of a planar (left) using the transfer matrix method [19] and a textured solar cell (right) using RCWA. In the left figure the measured quantum efficiency (squares) for a planar sample is shown and compared to the simulated absorption of a structure with similar setup (black line). Also shown is the simulated absorption in a sample with assumed Lambertian scattering (grey line). On the right hand side, the measured quantum efficiency (squares) for a random texture and the simulated absorption in the periodic texture ( line) are shown. 
Furthermore, we used the TMM to estimate the absorption in the Lambertian limit (grey line). For this purpose, the thickness of the silicon absorber layer was multiplied by $4 \mathrm{n}^{2}$ for each wavelength. Because for Lambertian scattering complete destruction of coherence effects can be expected, a wider thickness variation was carried out $\left(4 \mathrm{n}^{2} 150 \mathrm{~nm}\right.$ in 10 steps). Fabry Perot oscillations were mostly (but not completely) suppressed in this way. Otherwise the calculation was the same as before. In the Lambertian limit we find a photocurrent density of $\mathrm{j}_{\mathrm{ph} 600-900}=18.5 \mathrm{~mA} / \mathrm{cm}^{2}$ in the respective range, corresponding to an estimated short-circuit current density of about. $16.8 \mathrm{~mA} / \mathrm{cm}^{2}$.

On the right side of Figure 5, the results for a textured surface are shown. The squares correspond to the measured EQE of a cell deposited on the sample with a random, sputtered etched texture.

\section{SUMMARY AND DISCUSSION}

In this paper we investigated the performance of random and periodic structures for light trapping in thin-film microcrystalline silicon solar cells. The investigation was based on the AFM scan of a scattering texture made of sputtered and etched $\mathrm{ZnO}$ and on solar cell measurements performed for solar cells deposited onto this scattering structure. The AFM scan was directly used as an input for the optical simulation of the random texture. The absorption in silicon was calculated with a scalar scattering approach. We found good agreement between the calculated absorption and the measured external quantum efficiency for this structure, assuming an internal quantum efficiency of $90 \%$. In a further investigation of the scattering structure, we have mathematically scaled the height and the period of the random structure and used the same method as before to calculate the absorbed current in the solar cell. We found that, as expected,the higher the aspect ratio is, the higher is the absorption. . Furthermore, we found that the current is correlated roughly to the height / length ratio. Going to very high aspect ratios, scattering close to the Lambertian limit can be achieved with the investigated structure.

The investigation of the periodic structure is based on an analysis of typical shapes in the random structure. This analysis was performed to create a periodic structure that has a realistic shape; it can be assumed that similar shapes can be produced using a mask and an etching process similar to the one used to create the random structure. Subsequently, the absorption in a theoretical solar cell model on this periodic shape was calculated using the RCWA method. For the obtained periodic structure, absorption comparable to the one of the original random structure was found. An optimization of the periodic structure was performed by scaling period and height of the structure. This procedure is similar to the one used for the random structure, although the scaling rage was smaller. It was found that also for the periodic structure, a higher current is obtained for higher aspect ratio. A correlation of current to aspect ratio could, however, not be observed. As could be expected for periodic structures, maxima in current were found for specific parameter sets that depend mainly on the period of the structure. The best structure had a period of $\Lambda=534 \mathrm{~nm}$ and a depth of $d=277 \mathrm{~nm}$. For this structure the current in a $1.1 \mu \mathrm{m}$ thick solar cell was increased by $\Delta j_{p h}=1.6 \mathrm{~mA} / \mathrm{cm}^{2}$ or $15 \%$ relative in the spectral range between $600 \mathrm{~nm}$ and $900 \mathrm{~nm}$. In the investigated range, the best periodic structure performed better than a random structure with comparable parameters.

From the performed investigation several conclusions can be drawn:

- None of the investigated structures, even the optimized ones, resulted in an absorption enhancement exceeding the Lambertian limit. Even though it is in principle possible to outperform Lambertian light trapping with complex periodic structures, a comparable performance cannot be expected for the simpler yet potentially easier producible periodic structures investigated in this study. The best light trapping shown here was achieved for random structure with an extremely high roughness.

- Overall the performance of periodic and random structures with a similar shape seems to be comparable, at least in a certain parameter range. For the initial periodic and random structure absorptions were calculated that are very close to what can be expected from EQE measurements.

- From an optical point of view, the aspect ratio or roughness of a structure seems to be the most important feature for light trapping. A high aspect ratio or roughness seems beneficial. From an electrical point of view, however, too much roughness is detrimental, because thin-film solar cells are known to shunt on rough substrates. This could mark an advantage for periodic structures. For periodic structures, maxima (and minima) in absorption occur for very specific periods and, to a lesser extent, heights. Consequently, comparably high improvements can be obtained for periodic structures with a moderate adjustment in period and height. 
Further investigations will be performed in order to extend the investigated parameter range for periodic structures and to investigate the effect of multiple scattering surfaces for random structures.

\section{ACKNOWLEDGMENTS}

The authors would like to thank all colleagues from Fraunhofer ISE, IMT and SERIS that have contributed in one way or another to this work. SERIS is sponsored by the National University of Singapore and Singapore's National Research Foundation (NRF) through the Singapore Economic Development Board. The Fraunhofer project photonic-Si is acknowledged for financial support.

\section{REFERENCES}

[1] Yablonovitch, E. , "Statistical Ray Optics", J Opt Soc Am 72, 899-907 (1982).

[2] Dominé, D., Haug, F. J., Battaglia, C., and Ballif, C. , "Modeling of light scattering from micro- and nanotextured surfaces", J Appl Phys 107, 044504 (2010).

[3] Battaglia, C. et al, "Efficient light management scheme for thin-film silicon solar cells via transparent random nanostructures fabricated by nanoimprinting" Appl. Phys. Lett. 96, 213504 (2010).

[4] Sheng, P., Bloch, A. N., and Stepleman, R. S., "Wavelengthselective absorption enhancement in thin-film solar cells", Appl. Phys. Lett. 43, 579-582 (1983).

[5] Heine, C., and Morf, R, H, "Submicrometer gratings for solar energy applications", App. Opt. 34, 2476-2482 (1995).

[6] Zaidi, S. H., Gee, J. M., and Ruby, D. S., "Diffraction Grating Structures in Solar Cells", Proc. 28th IEEE Photovoltaic Specialists Conference, 395-398 (2000).

[7] Peters, M., Rüdiger, M., Hauser, H., Hermle, M., and Bläsi, B., "Diffractive gratings for crystalline silicon solar cells_optimum parameters and loss mechanisms", Prog. Photovolt. Res. App. (2011), DOI: 10.1002/pip.1151.

[8] Mellor, A., Tobias, I., Marti, A. Mendes, M. J., and Luque, A. "Upper limits to absorption enhancement in thick solar cells using diffraction gratings", Prog. Photovolt. Res. App. 19, 676 - 687 (2011).

[9] Kirchartz, T. [Physics of nanostructured solar cells], V. Badescu (Edt) Nova Science Publishers, 1-40 (2009).

[10] Yu, Z., Raman, A., and Fan, S., "Fundamental limit of light trapping in grating structures", Opt. Exp.18, 366380 (2010).

[11] Gjessing, J., Sudbo, A. S., and Marstein, E. S., "A novel back-side light trapping structure for thin silicon solar cells", J. Europ. Opt. Soc. 6, 11020 1-4. (2011).

[12] Peters, M. et al., "Optical Simulation of Silicon Thin-Film Solar Cells", proceedings of ICMAT (2011), to be published.

[13] Moharam, M. G., "Stable implementation of the rigorous coupled-wave analysis for surface-relief gratings: enhanced transmittance matrix approach" J. Opt. Soc. Am. A, 12, 1077 - 1086 (1995).

[14] Lalanne, P., and Jurek, M. P., "Computation of the near-field pattern with the coupled wavemethod for transverse magnetic polarization", J. Mod. Optics 45, 1357-1374 (1998).

[15] Elgamel, H. E. A., "High efficiency polycrystalline silicon solar cells using low temperature PECVD process", IEEE Transactions on Elec. Dev. 45, 2131 - 2137 (1998).

[16] A.V. Shah et al., "Thin-film Silicon Solar Cell Technology”, Prog. Photovolt: Res. Appl. 12, 113-142 (2004)

[17] Jäger, K., van Swaaij, R. A. C. M. M., Zeman, M, "A Full Scalar Scattering Model for Nano-Textured Interfaces", in "Optical Nanostructures and Advanced Materials for Photovoltaics" (Optical Society of America, 2011), PWC5

[18] Vet, B., Grancic, B., Isabella, O., Solntsev, S., and Zeman, M., "Optical and Electrical Simulations of Advanced Silicon Based Solar Cell Devices", Proceedings of the $24^{\text {th }}$ European Photovoltaic Solar Energy Conference $2682(2009)$

[19] Macleod, H.A., [Thin-Film Optical Filters], 3rd Ed. Institute of Physics Publishing,Bristol and Philadelphia, 141 (2001).

[20] Rockstuhl, C. et al., "Comparison and optimization of randomly textured surfaces in thin-film solar cells", Opt. Exp., 18, A335-A341 (2010) 